\title{
Reflets
}

Revue d'intervention sociale et communautaire

\section{La formation à l'offre active de services de santé et de services sociaux en français - La valeur ajoutée du Consortium national de formation en santé (CNFS)}

\section{CNFS}

Volume 20, numéro 2, automne 2014

URI : https://id.erudit.org/iderudit/1027589ar

DOI : https://doi.org/10.7202/1027589ar

Aller au sommaire du numéro

Éditeur(s)

Reflets, Revue d'intervention sociale et communautaire

ISSN

1203-4576 (imprimé)

1712-8498 (numérique)

Découvrir la revue

Citer cet article

CNFS (2014). La formation à l'offre active de services de santé et de services sociaux en français - La valeur ajoutée du Consortium national de formation en santé (CNFS). Reflets, 20(2), 154-165. https://doi.org/10.7202/1027589ar d'utilisation que vous pouvez consulter en ligne. 


\section{La formation à l'offre active de services de santé et de services sociaux en français - La valeur ajoutée du Consortium national de formation en santé (CNFS) ${ }^{1}$}

\section{CNFS}

\section{La mise en contexte}

Depuis sa création en 2003, le CNFS a pour mandat d'améliorer les services de santé en français pour les communautés de la francophonie hors Québec par la formation postsecondaire de professionnelles ou professionnels de la santé et des services sociaux francophones et par la recherche qui s'y rattache. En 2008 , le consultant responsable de l'évaluation sommative du CNFS formulait une recommandation importante pour contrer le décalage entre la formation offerte aux futurs professionnelles ou professionnels de la santé et la pratique dans les milieux de travail :

Il est recommandé que le CNFS et ses institutions membres prennent tous les moyens à leur disposition pour s'assurer que les personnes diplômées des programmes soutenus par le CNFS arrivent sur le marché du travail outillées pour faire face aux défis d'offrir et d'organiser des services de santé en français linguistiquement et 
culturellement appropriés en milieu minoritaire. [...] Ces diplômés sont appelés à devenir les artisans pour ne pas dire les fers de lance de l'amélioration des services de santé en français. (LeBlanc, 2008, p. 43)

À la suite de cette recommandation, le CNFS a réalisé deux projets sur la problématique en question : 1 . une recension des ouvrages, des pratiques et des outils existants; 2 . la tenue de rencontres auprès d'une centaine d'intervenantes ou intervenants clés de divers milieux et régions du pays (étudiantes ou étudiants, enseignantes ou enseignants, personnes œuvrant en santé, chercheuses ou chercheurs, gestionnaires et décideuses ou décideurs). De plus, une recherche effectuée en 2009 concluait notamment que les futurs professionnelles ou professionnels n'ont pas toujours l'occasion d'étudier pendant leur formation les effets des barrières linguistiques sur l'accès aux services de même que sur leur qualité (Bouchard etVézina, 2009). Les résultats des études menées par le CNFS et autres groupes de chercheuses ou de chercheurs (Bouchard et Vézina, 2009; Benoît, et collab., 2014 [accepté]) ont permis d'identifier les contenus et les outils de formation nécessaires pour sensibiliser et conscientiser les étudiantes et étudiants aux réalités des communautés francophones en contexte minoritaire et à l'importance de l'offre active des services de santé et des services sociaux en français.

Ainsi, appuyé par toute une équipe de collaborateurs chevronnés, le CNFS a lancé en janvier 2012 une première série de matériel imprimé (Bouchard et Savoie, 2011; Lortie, Lalonde et Bouchard, 2012) et audiovisuel (Consortium national de formation en santé, 2011).

Dans la lignée du travail effectué au cours des dernières années, le CNFS dévoilait lors de son Assemblée générale annuelle de juin 2013 l'énoncé d'engagement suivant cosigné par les dirigeantes et dirigeants de toutes ses institutions membres : 


\section{Énoncé d'engagement du CNFS envers la for- mation à l'offre active de services de santé et de services sociaux en français}

Le CNFS et ses institutions membres s'engagent à prendre tous les moyens à leur disposition pour s'assurer que les diplômés des programmes soutenus par le CNFS arrivent sur le marché du travail avec les connaissances et les compétences nécessaires, afin qu'ils soient en mesure, avec conviction et confiance, d'intervenir, d'appuyer et de bonifier les services de santé et services sociaux en français offerts en milieu minoritaire dans l'ensemble du pays. Pour ce faire, le CNFS et ses institutions membres mettront en place des activités concrètes visant à intégrer dans les programmes de formation des thématiques portant sur l'offre active.

\section{L'offre active de services de santé et de services sociaux en français}

\section{La définition de l'offre active}

Tel que présenté dans son Cadre de référence pour la formation à l'offre active des services de santé en français (2012), le CNFS définit de façon plus large l'offre active de services de santé et de services sociaux de qualité en français. Pour lui, l'offre active ne se limite pas simplement à des services dans la langue de la personne; elle doit aussi comprendre une approche globale de la planification et de la prestation des services de santé et des services sociaux destinés à une communauté en milieu minoritaire. Il s'agit d'une approche proactive qui tient compte de l'état de santé de la communauté et de son manque historique de services dans sa langue. Cette approche fait appel à la mobilisation de la communauté; elle tient compte de la diversité culturelle des personnes; elle s'inscrit dans 
une perspective éthique; elle met en place des mesures proactives d'intervention et elle vise à assurer la qualité des services et l'équité des bénéfices auxquels ont droit tous les francophones du Canada. En somme, pour le CNFS, « une offre active réussie est centrée sur la personne qui reçoit les soins ou les services. Elle reconnait et respecte la personne comme être unique avec toutes ses différences linguistiques, culturelles et autres " (Consortium national de formation en santé, 2012).

\section{Les fondements de l'offre active}

Au terme de la recherche effectuée pour réaliser son Cadre de référence pour la formation à l'offre active des services de santé en français (2012), le CNFS précise ainsi les fondements de l'offre active : "L'offre active des services de santé en français aux populations francophones vivant en situation minoritaire au Canada est une question de qualité, de sécurité, de légitimité et, de ce fait, une question d'éthique. "

Une des recherches (Castaño, et collab., 2007) démontre également que certains facteurs, tels la douleur, la vulnérabilité ou le stress, font souvent en sorte que les personnes, qui maitrisent pourtant bien le français et l'anglais dans d'autres circonstances, n'arrivent plus à s'exprimer adéquatement dans leur langue seconde. D'ailleurs, selon Sarah Bowen (2001), les barrières linguistiques engendrent des risques tant pour ces personnes que pour l'organisme qui leur offre des soins ou des services. En effet, selon Bowen, les problèmes de communication

- diminuent l'adhésion des personnes aux soins ou aux services;

- diminuent l'accès aux soins ou aux services préventifs;

- entraînent des diagnostics erronés ou des erreurs médicales;

- augmentent le nombre de tests ou de consultations médicales;

- ont des répercussions négatives sur la santé;

- entraînent des incidents critiques;

- réduisent la satisfaction des personnes, de leur famille et des fournisseurs de soins ou de services;

- augmentent les coûts des soins de santé. 
Des services de santé et des services sociaux de qualité dépendent non seulement de la capacité des professionnelles ou professionnels à maitriser des connaissances et des techniques précises, mais aussi de leurs habiletés à communiquer, car ils devront aider, conseiller, orienter et informer les usagers. La capacité de comprendre et d)être compris est donc essentielle à une relation efficace entre l'intervenante ou intervenant et la personne bénéficiaire de services de santé ou de services sociaux. Certains auteurs (Bowen, 2001; Langlois, 2008) précisent également que les barrières linguistiques augmentent le risque d'offrir des soins ou des services contraires à l'éthique. En fait, la communication devient le principal outil des professionnelles ou professionnels de la santé et des services sociaux ainsi qu'un préalable à la sécurité des soins ou des services qu'ils offrent (Schyve, 2007).

Dans son rapport intitulé Accès aux services de santé pour les populations insuffisamment servies au Canada, Sarah Bowen (2000) signale que quatre groupes au Canada sont confrontés à des difficultés d'accès en raison de leur langue : les Autochtones, les personnes immigrantes, les personnes Sourdes et, selon leur lieu de résidence, les personnes qui ne parlent qu'une seule des deux langues officielles du Canada. Au pays, près de deux millions de personnes vivent en situation minoritaire dans des communautés de langue officielle. Elles sont confrontées quotidiennement à des barrières linguistiques pour accéder à des services de santé ou à des services sociaux, à des programmes de promotion de la santé et du bien-être, ainsi qu'à des programmes de prévention des maladies.

À la lumière des études, il ressort que l'offre active et l'accès à des services de santé et à des services sociaux en français dans les communautés francophones en situation minoritaire (CFSM) est une question :

- de qualité des soins et services;

- de sécurité des patients ou bénéficiaires;

- d'éthique professionnelle;

- d'humanisation et d'efficacité des soins et services;

- de droits et d'équité;

- de culture organisationnelle;

- de sensibilisation de la population francophone; 
- de développement durable des communautés.

\section{Des pratiques exemplaires}

\section{La formation à l'offre active - La valeur ajoutée du CNFS}

Pour le CNFS, la formation à l'offre active de services de santé et de services sociaux en français vise avant tout à conscientiser les futurs professionnelles ou professionnels aux enjeux et aux défis liés à la prestation des services en français et à mieux les outiller pour intervenir avec conviction et confiance auprès des populations francophones vivant en contexte minoritaire.

Les institutions membres et les partenaires régionaux du CNFS ont toujours attaché une importance particulière à la formation de leaders et de catalyseurs de changement en faveur d'une offre accrue de services de santé et de services sociaux en français. L'intégration de la formation à l'offre active représente donc la valeur ajoutée du CNFS et permet un impact positif sur l'accès des francophones vivant en contexte minoritaire à des services de qualité dans leur langue.

Le CNFS a ainsi produit d'excellents outils qui visent principalement à bonifier la formation des étudiantes et des étudiants inscrits aux programmes en santé et en services sociaux soutenus par le CNFS. Ces outils se sont vite avérés tout aussi utiles pour les professionnelles ou professionnels et les gestionnaires de la santé et des services sociaux en poste. Disponibles en ligne (www.cnfs.net), ils favorisent l'acquisition de connaissances, de compétences et d'attitudes, afin de permettre aux futurs professionnelles ou professionnels et aux personnes déjà en poste d'intervenir avec conviction et confiance en milieu de travail et d'offrir activement des services en français de qualité dans les communautés francophones en situation minoritaire.

Le matériel produit à ce jour par le CNFS :

- Le Cadre de référence pour la formation à l'offre active des services de santé en français, publié en janvier 2012 et traduit en anglais en juillet 2012; 
- Le Cahier de réflexion et d'accompagnement et des vidéos présentant les témoignages de huit bénéficiaires francophones ou leur famille, publiés en janvier 2012;

- La Boîte à outils pour l'offre active, portail Web multimédia en ligne depuis novembre 2013, qui appuie la formation des futurs professionnelles ou professionnels à l'offre active des services sociaux et de santé en français et qui est aussi pertinente pour la formation continue des professionnelles ou professionnels et des gestionnaires en poste.

D'autres initiatives ont également été mises en œuvre par les institutions membres du CNFS, entre autres :

- des séances d'information sur l'offre active pour les enseignants et enseignantes et précepteurs de stages utilisant les outils pédagogiques et le matériel produits par le CNFS pour les appuyer;

- l'ajout de contenus pédagogiques sur l'offre active dans le curriculum des cours;

- l'ajout de contenus et de critères d'évaluation lors de la formation clinique et lors de formations continues.

Finalement, le 26 novembre 2013, le CNFS tenait une première rencontre nationale sur l'offre active dont le but principal était d'appuyer les intervenantes et intervenants pour l'intégration des concepts d'offre active dans les programmes de formation en santé et en services sociaux. Des intervenantes et intervenants de divers milieux et régions du Canada étaient présents, dont les coordonnateurs du CNFS des institutions membres, les partenaires régionaux du CNFS, des enseignantes ou enseignants, des chercheuses ou chercheurs et des représentantes ou représentants de Santé Canada. Plusieurs pistes d'action pour l'avancement de la formation, de la recherche et de la promotion de l'offre active ont été identifiées, notamment la mise sur pied prioritaire d'une formation pour les enseignants et enseignantes dans le but de les aider à intégrer les concepts de l'offre active dans les programmes de formation des professionnelles ou professionnels de la santé et des services sociaux. 


\section{La force et l'impact du CNFS}

Le CNFS est un modèle de collaboration interinstitutionnelle unique et efficient, et ce, à tous les échelons, tant national que provincial, territorial ou régional (Cardinal, Champagne et Eddie, 2012; 2013). La gouvernance et la structure organisationnelle du Consortium permettent d'assurer la cohérence, la synergie et la coopération entre ses institutions membres et ses partenaires régionaux, avec la coordination et le leadership efficaces du Secrétariat national. Les capacités et les forces du CNFS dépassent la somme de celles de ses membres.

Cette alliance stratégique exceptionnelle que représente le CNFS agit comme un effet de levier dans chaque institution membre et au sein des communautés desservies. Les retombées du CNFS sont nombreuses dans le domaine de la formation postsecondaire en santé et en services sociaux et se retrouvent également dans le dossier de l'offre active. Le modèle du CNFS favorise le partage des ressources, des connaissances, des expertises, des recherches et du matériel de formation et de promotion sur l'offre active. Ce partage permet ainsi une portée et un impact encore plus grands, non seulement au Canada, mais aussi à l'étranger, grâce à la technologie qui permet d'accéder aux documents et aux outils mis en ligne (Pays de Galles, États-Unis, pays francophones d'Afrique, etc.).

La coopération et la concertation interinstitutionnelles (institutions membres, partenaires régionaux et Secrétariat national) sont donc devenues le moteur et la marque de commerce du Consortium et contribuent grandement à sa cohésion et à son pouvoir d'attraction. De plus, les programmes de formation soutenus par le CNFS contribuent activement à la fierté et à l'identité des francophones, et ce, encore plus ces dernières années, soit depuis l'intégration de contenus et d'outils sur la formation à l'offre active. La contribution du CNFS et de ses membres est donc très importante pour assurer une offre active accrue de services de santé et de services sociaux en français dans l'ensemble du pays. 


\section{La vision d'avenir}

\section{Une approche globale}

L'offre active de services de santé et de services sociaux de qualité en français représente une approche globale en ce qui concerne la prestation des services aux francophones du Canada vivant en milieu minoritaire. À partir d'une approche centrée sur les personnes, sur leur dignité et leurs droits, l'offre active exige la responsabilisation collective du système de santé. L'offre active représente un acte professionnel éthique, un exercice de leadership transformationnel qui mobilise les ressources et les efforts de toutes les composantes du système de santé afin que l'ensemble de la population canadienne reçoive un traitement juste et équitable.

Les professionnelles ou professionnels jouent évidemment un rôle de premier plan dans la prestation de services de santé et de services sociaux. C'est pourquoi les programmes de formation, d'accompagnement et de perfectionnement doivent préparer les étudiantes ou étudiants dans ces domaines à agir avec confiance, conviction et compétence en vue d'une offre active de services de qualité en français dans leur milieu de travail.

\section{L'engagement du CNFS}

Pour sa part, le CNFS s'engage à poursuivre et à renforcer les initiatives de formation qu'il soutient selon un cadre d'apprentissage inspiré des thèmes priorisés dans le Rapport $d u$ Dialogue sur l'engagement des étudiants et des futurs professionnels pour de meilleurs services de santé en français dans un contexte minoritaire (Bouchard,Vézina et Savoie, 2010). Le but étant de former les futurs professionnelles ou professionnels de la santé et des services sociaux pour qu'ils agissent comme leaders dans leur milieu de travail en contexte minoritaire, par l'acquisition des savoirs suivants :

- les futurs professionnelles ou professionnels de la santé et des services sociaux devront

- acquérir des connaissances (savoir); 
- acquérir des compétences (savoir-faire);

- adopter des attitudes (savoir-être).

- les professionnelles ou professionnels diplômés de la santé et des services sociaux évolueront vers

- un leadership mobilisateur et transformateur (savoir vivre ensemble);

- un leadership éthique (savoir agir).

La sécurité des personnes qui reçoivent des soins de santé ou qui ont recours aux services sociaux est une priorité à l'échelle pancanadienne et internationale. Il s'avère donc essentiel d'intégrer l'offre de services dans la langue des bénéficiaires en tant que compétence dans le cadre de la formation des futurs professionnelles ou professionnels comme dans celui de la formation continue des professionnelles ou professionnels et des gestionnaires déjà en poste.

\section{Un défi de taille}

Les enjeux et défis sont nombreux et les gestionnaires comme les professionnelles ou professionnels dans les systèmes de santé ne sont pas outillés actuellement pour assurer une offre active de services de santé et de services sociaux en français qui soient culturellement et linguistiquement appropriés.

D'autre part, les gouvernements fédéral, provinciaux et territoriaux commencent à se pencher sur la question de l'offre active des services en français, non seulement dans le domaine de la santé, mais aussi dans d'autres secteurs aux besoins émergents, notamment celui de la justice (Cardinal, et collab., 2005; Jourdain, 2000).

Le concept de l'offre active étant assez nouveau, il est souvent méconnu des communautés et des diverses instances gouvernementales et institutionnelles, tout comme des fournisseurs de services et des professionnelles ou professionnels eux-mêmes. La tâche est colossale et le rôle du CNFS et de tous les partenaires et intervenantes ou intervenants concernés est donc primordial pour l'avancement de ce dossier qui en est un d'importance capitale. 


\section{Remerciements}

Le CNFS tient à remercier toutes les personnes qui ont contribué ces dernières années aux diverses initiatives qu'il a soutenues et mises en œuvre dans le domaine de l'offre active. Ces initiatives, tant sur le plan de la recherche que sur celui de la production et de la promotion de matériel, ont permis de mieux connaître et comprendre le concept et l'impact de l'offre active, mais aussi, de sensibiliser, informer et former les personnes concernées, tant au sein des institutions d'enseignement postsecondaire qu'au sein d'organismes et d'établissements de santé et de services sociaux.

\section{Note}

1 Le Consortium national de formation en santé (CNFS) est un regroupement pancanadien de onze institutions d'enseignement universitaire et collégial offrant des programmes de formation en français en santé et en services sociaux, et de six partenaires régionaux qui facilitent l'accès à ces programmes de formation. Le CNFS compte également un Secrétariat national, situé à Ottawa, qui joue un rôle de leadership et de coordination. Cette alliance stratégique permet d'accroitre la présence et l'apport de professionnelles ou professionnels et de chercheurs francophones et bilingues, afin d'améliorer l'offre de services de santé et de services sociaux en français de qualité et adaptés aux besoins des communautés francophones en situation minoritaire partout au pays. Les programmes et initiatives du CNFS sont rendus possibles grâce au financement de Santé Canada dans le cadre de la Feuille de route pour les langues officielles du Canada 2013-2018. Pour de plus amples renseignements sur le CNFS, visitez le site Internet : $\underline{\text { www.cnfs.net }}$

Consortium national de formation en santé, Secrétariat national

223, rue Main Ottawa (Ontario) K1S 1C4

Tél. : 613-244-7837 / Téléc. : 613-244-0699

\section{Bibliographie}

BENOÎT,Josée, et collab. (2014 [accepté]). «Les approches pédagogiques et les contenus de formation dans les programmes de santé et de service social en milieu minoritaire francophone au Canada ", Minorités linguistiques et sociétés.

BOUCHARD, Pier, et Mylène SAVOIE (2011). Document de réflexion pour les DVD «Quand la santé crest aussi la langue! ", [rapport], Consortium national de formation en santé, Ottawa, 28 p.

BOUCHARD, Pier, et SylvainVÉZINA (2009). L'outillage des étudiants et des nouveaux professionnels: un levier essentiel pour l'amélioration des services de santé en français, [rapport de recherche], Moncton, Consortium national de formation en santé, $66 \mathrm{p}$. 
BOUCHARD, Pier, SylvainVÉZINA et Mylène SAVOIE (2010). Rapport du Dialogue sur l'engagement des étudiants et des futurs professionnels pour de meilleurs services de santé en français dans un contexte minoritaire, [rapport de recherche], Consortium national de formation en santé, Ottawa, $37 \mathrm{p}$.

BOWEN, Sarah (2000). «Accès aux services de santé pour les populations insuffisamment servies au Canada ", dans Santé Canada, Certaines circonstances, [rapport de recherche], Ottawa, p.1-66.

BOWEN, Sarah (2001). Barrières linguistiques dans l'accès aux soins de santé, [rapport de recherche], Ottawa, Santé Canada, 141 p.

CARDINAL, Linda, Éric CHAMPAGNE et Marie-Hélène EDDIE (2012). La gouvernance du Consortium national de formation en santé : Engagement et résilience, [rapport de recherche], Ottawa, $48 \mathrm{p}$.

CARDINAL, Linda, Éric CHAMPAGNE et Marie-Hélène EDDIE (2013). « Nouvelle gouvernance publique et innovation : le cas du Consortium national de formation en santé ", Revue gouvernance, Vol. 10, No1, p. 1-14.

CARDINAL, Linda, et collab. (2005). Un état des lieux - les services en français dans le domaine de la justice en Ontario, [rapport de recherche], Chaire de recherche sur la francophonie et les politiques publiques, Ottawa, $127 \mathrm{p}$.

CASTAÑO, M.Teresa, et collab. (2007). "Challenges of providing mental health services in Spanish ", Professional Psychology: Research and Practice, Vol. 38, No 6, p. 667-673.

CONSORTIUM NATIONAL DE FORMATION EN SANTÉ (2011). Quand la santé c'est aussi la langue, réf. du 30 mai 2014, http://cnfs.net/fr/vidlist.php?tmpl=7\&ext=7

JOURDAIN, Guy (2000). «Le groupe de travail sur lramélioration des services en français au sein du système judiciaire du Manitoba : outil efficace pour faciliter le long périple menant de la parole aux actes en matière dradministration de la justice dans les deux langues officielles ", Revue de la common law, Vol. 3, № 1 et 2, p. 67 à 86.

LANGLOIS, Lyse (2008). Anatomie du leadership éthique : pour diriger nos organisations d'une manière consciente et authentique, Les Presses de l'Université Laval, $138 \mathrm{p}$.

LEBLANC, Pierre (2008). Rapport de l'évaluation sommative du Projet de formation et de recherche du Consortium national de formation en santé, [rapport d'évaluation], Ottawa, 209 p.

LORTIE, Lise, André J. LALONDE et Pier BOUCHARD (2012). Cadre de référence pour la formation à l'offre active des services de santé en français [rapport], Consortium national de formation en santé, Ottawa, 27 p.

SCHYVE, Paul M. (2007). «Language differences as a barrier to quality and safety in health care: the Joint Commission perspective ", Journal of General Internal Medicine,Vol. 22, (suppl. 2), p. 360-361. 